\title{
Supratentorial cavernous malformations adjacent to the corticospinal tract: surgical outcomes and predictive value of diffusion tensor imaging findings
}

\author{
${ }^{*}$ Yuanxiang Lin, MD, ${ }^{1}$ Fuxin Lin, MD, ${ }^{1}$ Dezhi Kang, MD, ${ }^{1}$ Yuming Jiao, MD, ${ }^{2-5}$ Yong Cao, MD, ${ }^{2-5}$ and \\ Shuo Wang, MD2-5 \\ 1Department of Neurosurgery, The First Affiliated Hospital of Fujian Medical University, Fujian Medical University, Fujian \\ Province; ' ${ }^{2}$ epartment of Neurosurgery, Beijing Tiantan Hospital, Capital Medical University, Beijing; ${ }^{3}$ China National Clinical \\ Research Center for Neurological Diseases, Beijing; ${ }^{4}$ Center of Stroke, Beijing Institute for Brain Disorders, Beijing; and ${ }^{5}$ Beijing \\ Key Laboratory of Translational Medicine for Cerebrovascular Disease, Beijing, People's Republic of China
}

OBJECTIVE Diffusion tensor imaging (DTI) findings may facilitate clinical decision making in patients with supratentorial cavernous malformations adjacent to the corticospinal tract (CST-CMs). The objective of this study was to determine the predictive value of preoperative DTI findings for surgical outcomes in patients with CST-CMs.

METHODS A prospectively maintained database of patients with CM referred to the authors' hospital between September 2012 and October 2015 was reviewed to identify all consecutive surgically treated patients with CST-CM. All patients had undergone sagittal T1-weighted anatomical imaging and DTI before surgery. Both DTI findings and clinical characteristics of the patients and lesions were analyzed with respect to surgery-related motor deficits. DTI findings included lesion-to-CST distance (LCD) and the alteration (i.e., deviation, interruption, or degeneration due to the CM) of CST on preoperative DTI images. Surgery-related motor deficits at 1 week and the last clinic visit ( $\geq 3$ months) after surgery were defined as short-term and long-term deficits, respectively. Preoperative and final modified Rankin Scale scores were also analyzed to identify the surgical outcomes in these patients.

RESULTS A total of 56 patients with 56 CST-CMs were included in this study. The mean LCD was $3.9 \pm 3.2 \mathrm{~mm}$, and alterations of the CST were detected in $20(36.7 \%)$ patients. One week after surgery, $21(37.5 \%)$ patients had short-term surgery-related motor deficits, but only $14(25.0 \%)$ patients had long term deficits at the last clinical visit. The mean patient follow-up was $14.7 \pm 10.1$ months. The difference between preoperative and final modified Rankin Scale scores was not statistically significant $(p=0.490)$. Multivariate analysis showed that both short-term $(p<0.001)$ and long-term ( $p$ $=0.002$ ) surgery-related motor deficits were significantly associated with LCD. Receiver operating characteristic (ROC) curve results were as follows: for short-term surgery-related motor deficits, the area under the ROC curve (AUC) was 0.860 , and the cutoff point was $L C D=2.55 \mathrm{~mm}$; for long-term deficits, the AUC was 0.894 , and the cutoff point was LCD $=2.30 \mathrm{~mm}$. Both univariate $(p=0.012)$ and multivariate $(p=0.049)$ analyses revealed that CST alteration on preoperative DTI was significantly correlated with short-term surgery-related motor deficits. On univariate analysis, deep location of the CST-CMs was significantly correlated with long-term motor deficits $(p=0.016)$. Deep location of the CST-CMs had a trend toward significance with long-term motor deficits on the multivariate analysis $(p=0.060)$.

CONCLUSIONS To facilitate clinical practice, the authors propose that $3.00 \mathrm{~mm}(2.55$ to $\sim 3.00 \mathrm{~mm})$ may be the safe LCD for surgery in patients with CST-CMs. A CST alteration on preoperative DTI and a deep location of the CST-CM may be risk factors for short- and long-term surgery-related motor deficits, respectively. A randomized controlled trial is needed to demonstrate the predictive value of preoperative DTI findings on surgical outcomes in patients with CST-CMs in future studies.

https://thejns.org/doi/abs/10.3171/2016.10.JNS161179

KEY WORDS diffusion tensor imaging; cavernous malformations; corticospinal tract; surgery; risk factor; vascular disorders

ABBREVIATIONS AUC = area under the ROC; aVDA = associated developmental venous anomaly; $\mathrm{AVM}=$ arteriovenous malformation; $\mathrm{CAO}=$ the corridor formed by the CST, arcuate fasciculus, and optic radiation; $\mathrm{CM}=$ cavernous malformation; $\mathrm{CST}=$ cortical spinal tract; $\mathrm{CST}-\mathrm{CM}=\mathrm{CM}$ adjacent to the CST; $\mathrm{DTI}=$ diffusion tensor imaging; $\mathrm{LCD}=$ lesion-to-CST distance; $\mathrm{mRS}=$ modified Rankin Scale; $\mathrm{ROC}=$ receiver operating characteristic.

SUBMITTED May 8, 2016. ACCEPTED October 13, 2016

INCLUDE WHEN CITING Published online March 31, 2017; DOI: 10.3171/2016.10.JNS161179

* Drs. Y. Lin and F. Lin contributed equally to this work. 


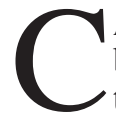
AVERNOUS malformations (CMs) are histologically benign vascular lesions consisting of irregular thin-walled sinusoidal vascular channels without interposed brain tissue. ${ }^{35}$ The incidence of CMs is approximately $0.4 \%-0.8 \%$ of the general population according to autopsy or MRI studies, and the supratentorial location is represented in almost $70 \%-80 \%$ of intracranial cases. ${ }^{10}$ Although there is general agreement on the surgical treatment for supratentorial CMs located in noneloquent areas, some controversy exists regarding the management of supratentorial CMs located in eloquent or deep areas. ${ }^{11}$ Especially for CMs adjacent to the corticospinal tract (CST$\mathrm{CMs}$ ), inadvertent trauma to the corticospinal tract (CST) may lead to devastating surgical outcomes. Thus, surgery of CST-CMs should be carefully weighed against the risk of hemorrhage. ${ }^{35}$ However, lack of white matter anatomical landmarks, distortion caused by lesions, and displacement of the fiber tracts make it difficult to identify the CST location and to evaluate the surgical risks before surgery.

Diffusion tensor imaging (DTI) tractography is the unique method that involves noninvasive tracking of the white matter pathways of the brain in vivo. ${ }^{27}$ The feasibility of DTI tractography has been determined for various intracranial lesions, $4,18,22,28$ including in the context of CMs. ${ }^{8}$ Preoperative fiber tracking is useful in planning surgical procedures involving deep-seated lesions adjacent to the CST and may help to avoid postoperative morbidity due to CST injury. ${ }^{27}$ Recently, the utility of DTI tractography in the resection of brainstem CMs has been reported. ${ }^{15}$ However, with only isolated case reports and small series describing its utility for supratentorial CMs, evidence of the predictive value that DTI tractography has on the outcomes of CST-CMs surgery is pending. ${ }^{1,6,21,27}$ Informed by our efforts to determine the predictive effect of preoperative functional findings for surgical outcomes of arteriovenous malformations (AVMs), ${ }^{26}$ we hypothesized that preoperative DTI findings might predict surgical outcomes and facilitate clinical decision making in patients with CST-CMs. Thus, we reviewed our prospectively maintained database of patients with CMs who were referred to our institution in order to identify the risk factors of postoperative motor deficits and to determine the predictive value of preoperative DTI findings for surgical outcomes in patients with CST-CMs.

\section{Methods \\ Patient Population}

A prospectively maintained database of $\mathrm{CM}$ patients who were referred to the Beijing Tiantan Hospital (China National Clinical Research Center for Neurological Diseases) between September 2012 and October 2015 was reviewed to identify all consecutive surgically treated patients with a CST-CM. Inclusion criteria were as follows: 1) a definitive diagnosis of CM determined by postoperative pathological results; 2) DTI examination performed before surgery; 3) lesion-to-CST distance (LCD) within $10 \mathrm{~mm}$ on preoperative DTI images; 4) surgical treatment of the CST-CM; 5) at least 3 months of follow-up; and 6) informed consent. This study was approved by the institutional review board of the Beijing Tiantan Hospital
Capital Medical University. Written informed consent was obtained from all patients or their guardians when they were enrolled in our CM database.

\section{Neuroimaging and Tractography}

The details of preoperative neuroimages and tractography have been shown in our prior studies. ${ }^{24,25}$ Conventional brain MRI and CT studies were obtained as part of the diagnostic workup. The sagittal T1-weighted anatomical MR images and DT images were acquired using a 3.0T MR system (SIEMENS Trio). The sagittal T1-weighted anatomical MR images were acquired using a gradientecho sequence with the following parameters: TR 2300 msec, TE $2.98 \mathrm{msec}$, slice thickness $1 \mathrm{~mm}, 176$ slices, FOV $256 \mathrm{~mm}$, flip angle $9^{\circ}$, matrix $64 \times 64$, voxel size $1 \times$ $1 \times 1 \mathrm{~mm}$, and a bandwidth of 240. The DTI studies were acquired using the diffusion-weighted echo-planar imaging technique with the following settings: TR $6100 \mathrm{msec}$, TE $93 \mathrm{msec}$, slice thickness $3 \mathrm{~mm}$, 45 slices, FOV $230 \times$ $230 \mathrm{~mm}$, matrix $128 \times 128$, and a motion-probing gradient in 30 orientations.

The generated image sets were processed on the iPlan 3.0 workstation (Brainlab). All image sets were automatically coregistered with each other and fused to the anatomical images using an automatic rigid registration. To track the CST, we used 2 regions of interest delineated in the precentral gyrus (seed) and pons (target). ${ }^{7}$ We selected a default fractional anisotropy threshold of 0.20 and a minimum fiber length of $70 \mathrm{~mm}$. Two neuroradiologists, with consensus, documented the locations of the regions of interest and the tracked fibers. They were blinded to the clinical information of the patients. The processed data sets were then incorporated into the neuronavigation platform for intraoperative navigation.

\section{Surgery}

The surgical principles for CMs located in critical areas were presented in our prior study ${ }^{24}$ Incidentally discovered CMs were treated conservatively. As radiosurgery has not shown proven benefit for patients with CMs, we do not offer it at our hospital. Patients with intractable seizures, severe or repeated hemorrhage, progressive neurological deficits, and severe headache were recommended for surgical treatment. The operations were performed by the senior authors (S.W. and Y.C.). The surgical approaches were chosen according to the excellent experience provided by Chang et al. ${ }^{9}$ and the location of the CST on preoperative DTI. The major principles were maximizing subarachnoid dissection and minimizing brain transgression. Associated venous anomalies and the hemosiderin rim were spared during surgery. Surgical adjuncts, including intraoperative monitoring of the evoked potentials, subcortical electrical stimulation, and a neuronavigation system, were used to localize the lesions and protect the eloquent brain tissues. The removed specimen was sent for pathological examination. Subcortical electrical stimulation was performed at the resection cavity wall nearest to the CST based on neuronavigation results, and sites with positive results were recorded. The intensity was initiated at $10 \mathrm{~mA}$ and increased stepwise in $2-\mathrm{mA}$ increments to 
a maximum of $16 \mathrm{~mA}$. Electroencephalographic mapping was used in patients who presented with epilepsy. Conventional MRI was performed 5 days later to confirm the radical obliteration.

\section{Variables}

Clinical characteristics of the patients, including age, sex, presenting symptom, number of preoperative hemorrhages caused by CST-CM, interval between the last hemorrhage and surgery, and history of radiosurgery, were collected. A hemorrhagic episode was defined as a sudden onset or worsening of a clinical neurological deficit, along with evidence of a new hemorrhage on emergent $\mathrm{CT}$ or MRI scans. Variables from each CST-CM, such as the side of the lesional hemisphere, largest diameter (including the hematoma), hematoma, surrounding edema, associated developmental venous anomaly (aDVA), and anatomical location of the CST-CM, were determined from preoperative CT and MRI scans. Based on the anatomical location, the lesions were categorized into 4 groups as determined by where the CM appeared relative to the CST: 1) cortical CM; 2) subcortical CM; 3) corona radiata CM; and 4) $\mathrm{CM}$ involving the posterior limb of the internal capsule. ${ }^{24}$ Cortical and subcortical CMs were defined as superficial CST-CMs. Conversely, corona radiata CMs and CMs involving the posterior limb of the internal capsule were considered deep CST-CMs (Figs. 1 and 2). Multiple CMs were documented in 1 patient. DTI findings including LCD and alteration of the CST were preoperatively docu- mented. Sections for LCD measurement were manually selected where the tracked fiber appeared to be nearest to the CM margin on sagittal T1-weighted anatomical images, but not to the margin of the hematoma or hemosiderin rim (Figs. 1 and 2). Alterations in the trajectory and/ or size of the fiber tract compared with the contralesional CST were qualitatively identified. Descriptions of the tract alteration patterns included "deviation," "interruption" (partial or complete), and "degeneration." Motor deficits of the contralesional limbs and modified Rankin Scale (mRS) score of the patients were documented 1 day before surgery. Complete resection of the CST-CM was documented on postoperative MRI or CT scans (Figs. 3 and 4). Postoperative complications, except for motor deficits, were recorded. Surgery-related motor deficit assessments were performed 1 week after surgery (short-term deficits), and at the last clinical visit (long-term deficits). All patients had a follow-up period that exceeded 3 months. A surgery-related motor deficit was defined as a new-onset or worsening of a deficit in muscle strength, ataxia, muscle tension, and deep sensory function after surgery. The mRS score was also documented at the last clinical visits (final mRS score). A final mRS score of 0-2 was considered a good outcome, and a final mRS score greater than 2 was considered a poor outcome.

\section{Statistical Analysis}

Data are reported as the mean and SD for continuous variables or frequency for categorical variables. An inde-
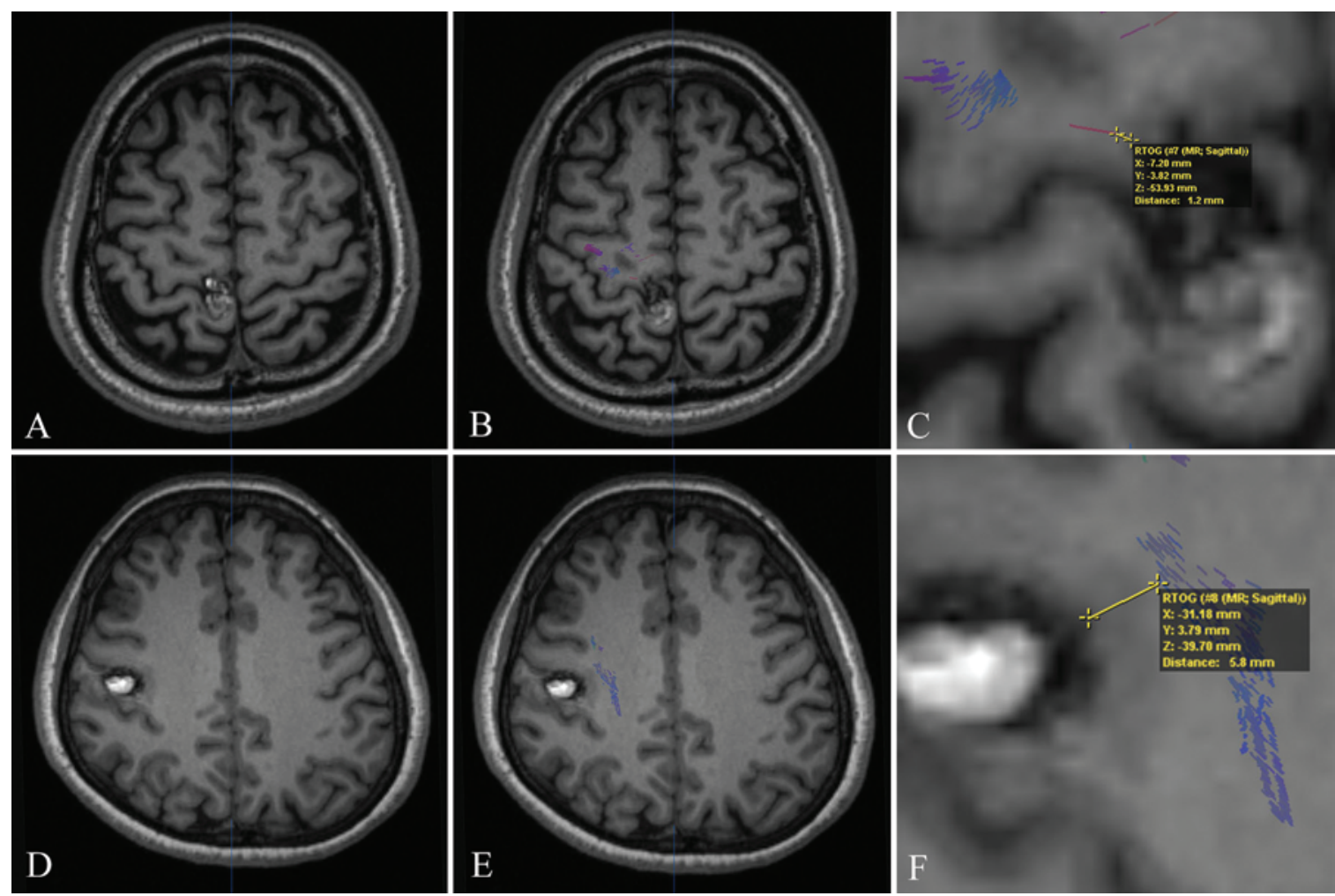

FIG. 1. CMs adjacent to the CST at the cortical (A) and subcortical (D) levels. DTI tractography was performed (B and E), and the LCD was measured ( $C$ and $F$ [enlarged areas from panels $A$ and $D$ ]) before surgery. Sections for LCD measurement were manually selected where the tracked fiber appeared to be nearest to the margin of the CM on sagittal T1-weighted anatomical images, but not to the margin of the hematoma or hemosiderin rim. Figure is available in color online only. 

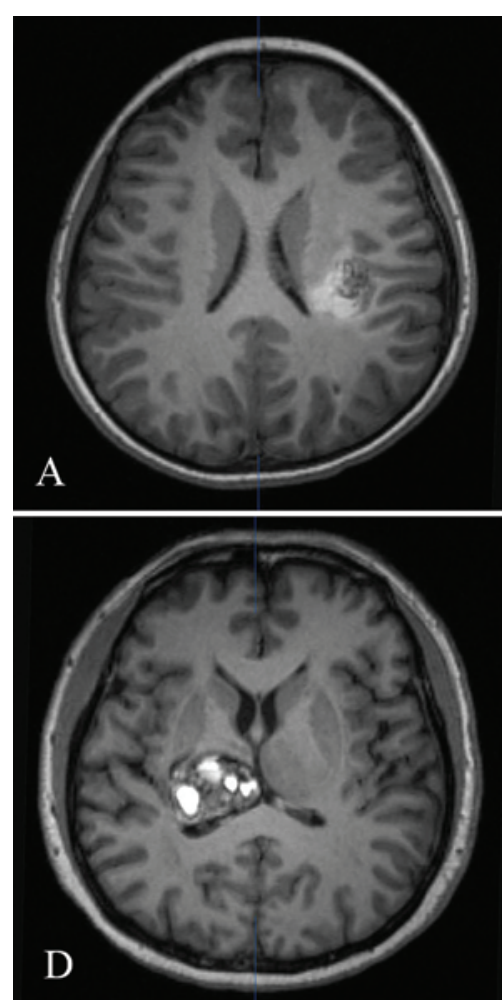
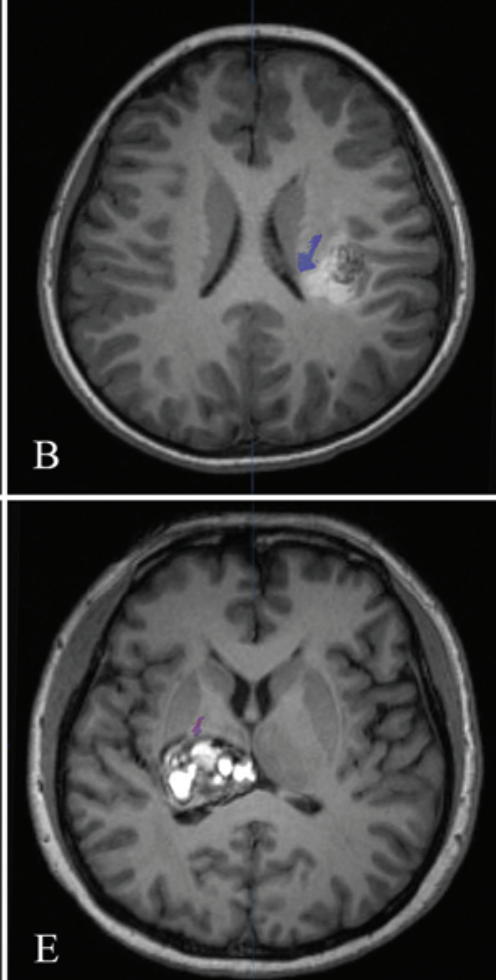
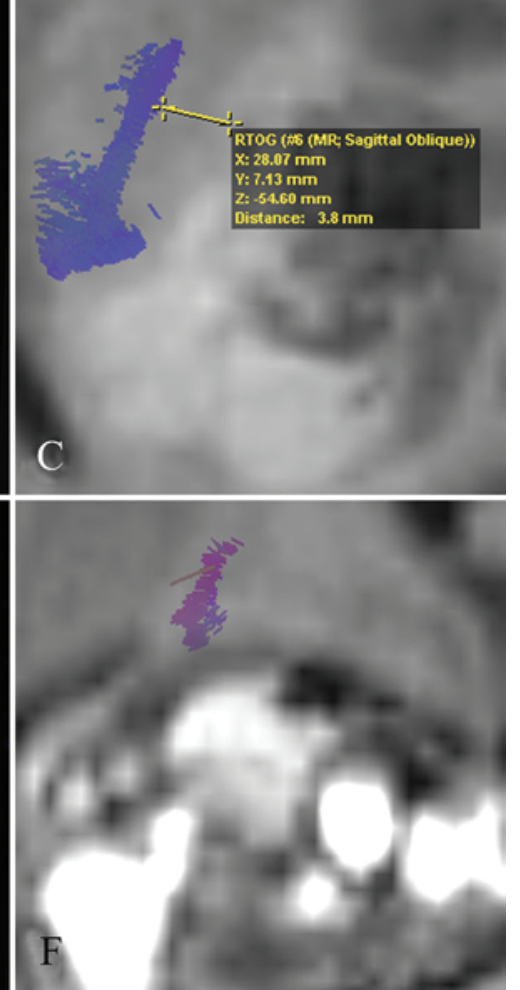

FIG. 2. Axial T1-weighted anatomical images showing CMs adjacent to the CST at the levels of the corona radiata (A) and the posterior limb of internal capsule (D). DTI tractography was performed (B and E), and the LCD was measured (C and F) before surgery. Sections for $L C D$ measurement were manually selected where the tracked fiber appeared to be nearest to the margin of the CM on sagittal T1-weighted anatomical images, but not to the margin of the hematoma or hemosiderin rim. Figure is available in color online only.
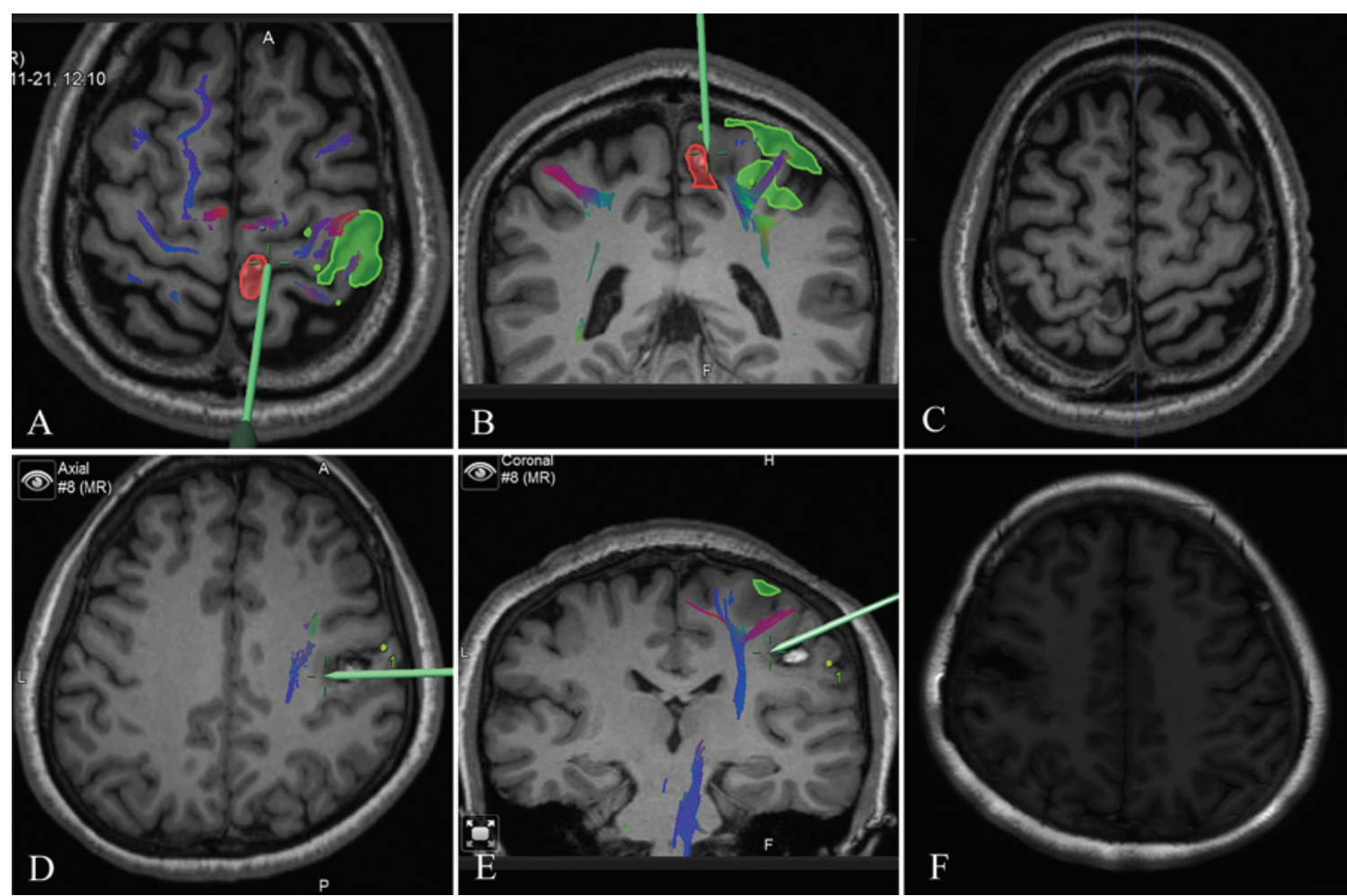

FIG. 3. DTI tractography images showing CMs adjacent to the CST at the cortical (A and B) and subcortical (D and E) levels. Intraoperatively, the navigational pointer identified the surgical extent in the same patients whose images are featured in Fig. 1. Postoperative MRI scans showing that the CMs were radically removed ( $\mathbf{C}$ and $\mathbf{F})$. Figure is available in color online only. 

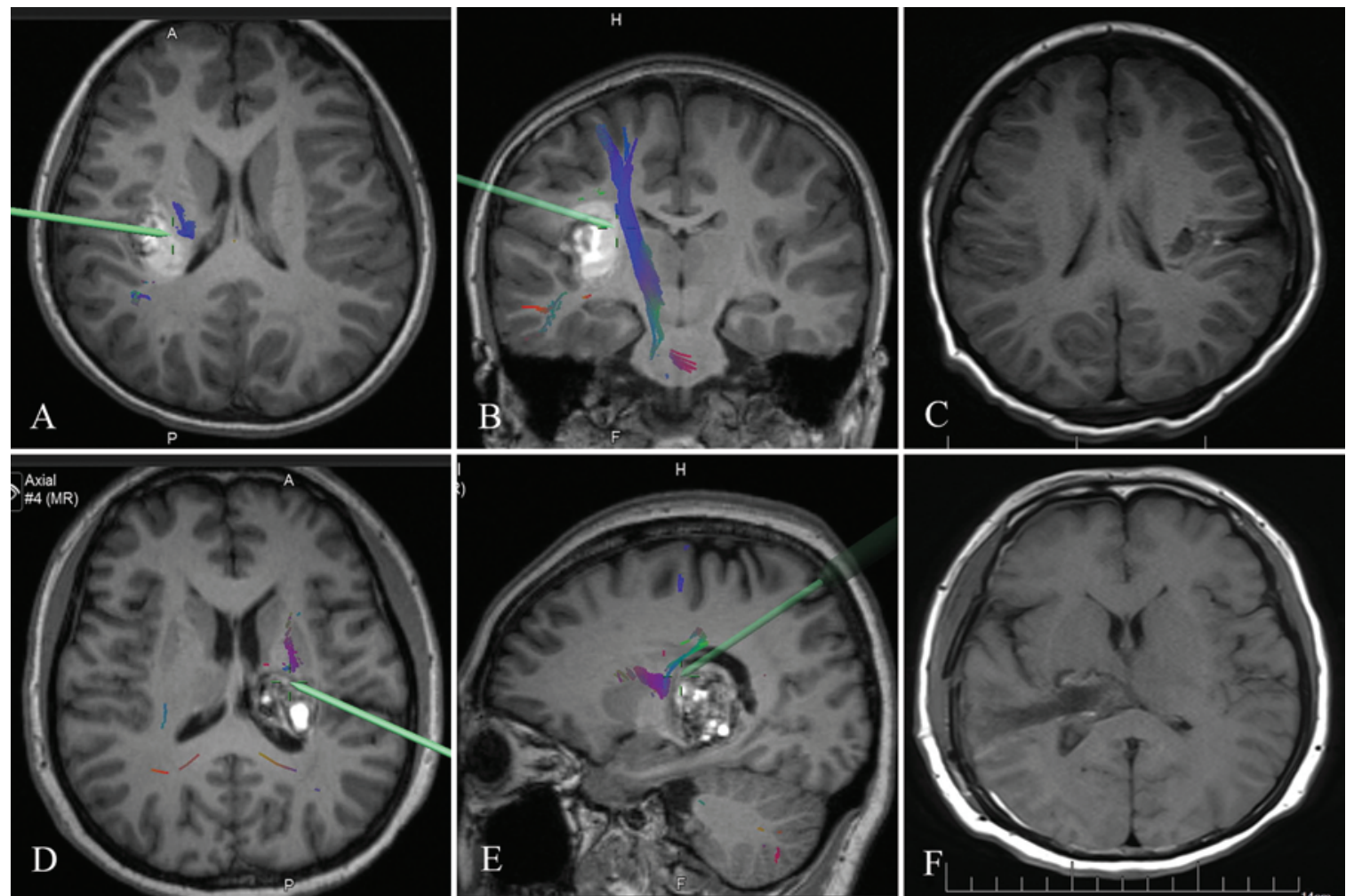

FIG. 4. DTI tractography images showing CMs adjacent to the CST at the levels of the corona radiata (A and B) and the posterior limb of internal capsule ( $\mathbf{D}$ and $\mathbf{E})$. Intraoperatively, the navigational pointer identified the surgical extent in the same patients whose images are featured in Fig. 2. Postoperative MRI scans showing that the CMs were radically removed ( $\mathbf{C}$ and $\mathbf{F}$ ). Figure is available in color online only.

pendent sample t-test was used for continuous variables. The chi-square test or Fisher exact test was used for dichotomized variables. Preoperative and final mRS scores were analyzed to identify the surgical outcomes in patients with CST-CM. Univariate and multivariate analyses were used to determine the relationship between the variables and surgery-related motor deficits. Variables with $p<0.1$ in the univariate analysis were then used in the multivariate analysis model. For the significant continuous variables, the receiver operating characteristic (ROC) curve analysis was applied to determine the predictive value and cutoff point. Statistical tests were considered significant at $\mathrm{p}<0.05$. Odds ratios are presented with $95 \%$ confidence intervals. Statistical analysis was performed using the statistical package SPSS (version 20.0.0, IBM Corp.).

\section{Results}

\section{Clinical Characteristics of the Patients}

A total of 56 patients with 56 CST-CMs were included in this study (Table 1). There were 38 (67.9\%) male patients and $18(32.1 \%)$ female patients, with a mean age of $28.5 \pm$ 12.1 years. The most common presenting symptoms were intracranial hemorrhage $(58.9 \%)$, seizure $(28.6 \%)$, functional deficits $(8.9 \%)$, and severe headache (3.6\%). Thirtythree $(58.9 \%)$ patients had preoperative hemorrhages; 6 $(10.7 \%)$ of these patients had 2 hemorrhages, and $1(1.8 \%)$ patient experienced 3 preoperative hemorrhages. Assuming that CMs are congenital with a uniform hemorrhage risk throughout life, the annual retrospective hemorrhage rate was $2.57 \%$ in this study. Three (5.4\%) patients had undergone radiosurgery before they were referred to our hospital. The mean lesion diameter was $29.4 \pm 11.0 \mathrm{~mm}$, including the size of the existent hematoma in 19 (33.9\%) patients. Surrounding edema and aDVA were detected in $17(30.4 \%)$ and 22 (39.3\%) patients, respectively. Twentysix (46.4\%) patients had superficial CST-CMs: 13 (23.2\%) cortical CST-CMs and 13 (23.2\%) subcortical CST-CMs. Thirty (53.6\%) patients had deep-seated CST-CMs; in 14 (25.0\%) of these patients the CST-CM was located at the corona radiata and in $16(28.6 \%)$ it involved the posterior limb of the internal capsule (Table 2). The mean LCD value was $3.9 \pm 3.2 \mathrm{~mm}$, and alterations of the CST were detected in $20(35.7 \%)$ patients. In 1 patient (1.8\%), the ipsilesional CST was not tracked because of the obvious edema surrounding the CM and the large associated hematoma. According to preoperative functional examination, 15 (26.8\%) patients had preoperative motor deficits; the mean mRS score was $1.21 \pm 0.8$ in these patients.

\section{Surgical Approaches and Surgical Outcomes}

Cortical CST-CMs were identified and then removed by separating the lesions from the surrounding brain tissue (Table 2 and Fig. 3). Five (38.5\%) of the subcortical CST-CMs were resected by transgressing the premotor or parietal cortex (transcortical approach), and the other 8 (61.5\%) subcortical CST-CMs were removed through the 
TABLE 1. Demographic and clinical characteristics of the patients with CMs adjacent to the CST

\begin{tabular}{|c|c|}
\hline Variable & Value \\
\hline No. of patients & 56 \\
\hline Mean age (yrs) & $28.5 \pm 12.1$ \\
\hline \multicolumn{2}{|l|}{ Sex } \\
\hline Male & $38(67.9)$ \\
\hline Female & $18(32.1)$ \\
\hline \multicolumn{2}{|l|}{ Presenting symptoms } \\
\hline Hemorrhage & $33(58.9)$ \\
\hline Seizure & $16(28.6)$ \\
\hline Headache & $2(3.6)$ \\
\hline Functional deficits & $5(8.9)$ \\
\hline \multicolumn{2}{|l|}{ No. of hemorrhages* } \\
\hline 0 & $23(41.1)$ \\
\hline 1 & $26(46.4)$ \\
\hline 2 & $6(10.7)$ \\
\hline 3 & $1(1.8)$ \\
\hline Mean ILHS (days) & $71.0 \pm 51.2$ \\
\hline \multicolumn{2}{|l|}{ Previous radiosurgery } \\
\hline Yes & $3(5.4)$ \\
\hline No & $53(94.6)$ \\
\hline \multicolumn{2}{|l|}{ Side } \\
\hline Left & $28(50.0)$ \\
\hline Right & $28(50.0)$ \\
\hline Mean diameter $(\mathrm{mm}) \dagger$ & $29.4 \pm 11.0$ \\
\hline \multicolumn{2}{|l|}{ Hematoma } \\
\hline Yes & $19(33.9)$ \\
\hline No & $37(66.1)$ \\
\hline \multicolumn{2}{|l|}{ Surrounding edema } \\
\hline Yes & $17(30.4)$ \\
\hline No & $39(69.6)$ \\
\hline \multicolumn{2}{|l|}{ Multiple CMs } \\
\hline Yes & $7(12.5)$ \\
\hline No & $49(87.5)$ \\
\hline \multicolumn{2}{|l|}{ aDVA } \\
\hline Yes & $22(39.3)$ \\
\hline No & $34(60.7)$ \\
\hline Mean preop mRS score & $1.21 \pm 0.8$ \\
\hline \multicolumn{2}{|l|}{ Preop motor deficit } \\
\hline Yes & $15(26.8)$ \\
\hline No & 41 (73.2) \\
\hline \multicolumn{2}{|l|}{ Location } \\
\hline Deep & $31(55.4)$ \\
\hline Superficial & $25(44.6)$ \\
\hline Mean LCD & $3.9 \pm 3.2$ \\
\hline \multicolumn{2}{|l|}{ CST alteration $\ddagger$} \\
\hline Yes & $20(35.7)$ \\
\hline No & $36(64.3)$ \\
\hline \multicolumn{2}{|l|}{ Positive sites of SCES } \\
\hline Yes & $50(89.3)$ \\
\hline No & $6(10.7)$ \\
\hline \multicolumn{2}{|l|}{ Complete resection } \\
\hline Yes & $55(98.2)$ \\
\hline No & $1(1.8)$ \\
\hline
\end{tabular}

CONTINUED IN NEXT COLUMN 》
» CONTINUED FROM PREVIOUS COLUMN

TABLE 1. Demographic and clinical characteristics of the patients with CMs adjacent to the CST

\begin{tabular}{cc}
\hline \multicolumn{1}{c}{ Variable } & Value \\
\hline Complications $\S$ & $52(92.9)$ \\
None & $3(5.4)$ \\
Intracranial infection & $1(1.8)$ \\
Seizure & \\
\hline S-MD1 & $21(37.5)$ \\
Yes & $35(62.5)$ \\
No & $14.7 \pm 10.1$ \\
\hline Follow-up (mos) & $1.1 \pm 1.2$ \\
\hline Mean final mRS & \\
\hline Surgical outcomes & $48(85.7)$ \\
Good & $8(14.3)$ \\
Poor & $14(25.0)$ \\
\hline S-MD2 & $42(75.0)$ \\
Yes &
\end{tabular}

ILHS = interval between the last hemorrhage and surgery; S-MD1 = motor deficits at 7 days after surgery; S-MD2 = surgery-related motor deficits at the last clinical visit; SCES = subcortical electrical stimulation.

Values represent the number of patients (\%) unless otherwise stated. Mean values are presented as the mean \pm SD.

* Before surgical treatment.

$\dagger$ The largest diameter of the lesion, including hematoma, on sagittal anatomical T1-weighted images.

$\ddagger$ The alteration in size and/or trajectory of the CST compared with that of the contralesional CST.

$\S$ Before discharge except for motor deficits.

pre- or postcentral sulcus (transsulcal approach) with the aid of neuronavigation (Fig. 3). ${ }^{9}$ For CST-CMs located at the corona radiata, both transcortical $(5,35.7 \%)$ and transsulcal approaches $(5,35.7 \%)$ were used. The approach opening the sylvian fissure and then through the corridor formed by the CST, arcuate fasciculus, and optic radiation based on intraoperative DTI navigation findings (CAO approach) ${ }^{24}$ was performed in $3(21.4 \%)$ patients with CSTCMs located at the lower corona radiata level (Fig. 4). In the remaining patient with a corona radiata CST-CM close to the midline, a posterior interhemispheric approach was performed. ${ }^{9}$ The CAO approach was used in 14 (87.5\%)

TABLE 2. Anatomical locations and surgical approaches of CMs

\begin{tabular}{ccccc}
\hline \multirow{2}{*}{$\begin{array}{c}\text { Surgical } \\
\text { Approach }\end{array}$} & \multicolumn{4}{c}{ CM Location } \\
\cline { 2 - 5 } & Cortex & Subcortex & CR & PLIC \\
\hline TC & $13(100.0)$ & $5(38.5)$ & $5(35.7)$ & $2(12.5)$ \\
\hline TS & $0(0.0)$ & $8(61.5)$ & $5(35.7)$ & $0(0.0)$ \\
\hline CAO & $0(0.0)$ & $0(0.0)$ & $3(21.4)$ & $14(87.5)$ \\
\hline PIA & $0(0.0)$ & $0(0.0)$ & $1(7.1)$ & $0(0.0)$ \\
\hline Total & $13(23.2)$ & $13(23.2)$ & $14(25.0)$ & $16(28.6)$ \\
\hline
\end{tabular}

$\mathrm{CR}=$ corona radiata level; $\mathrm{PIA}=$ posterior interhemispheric approach; $\mathrm{PLIC}=$ posterior limb of the internal capsule level; $\mathrm{TC}=$ transcortical approach; $\mathrm{TS}=$ transsulcal approach.

Percentages for total values are based on the total of 56 patients. Percentages for all other values are based on the column totals. 

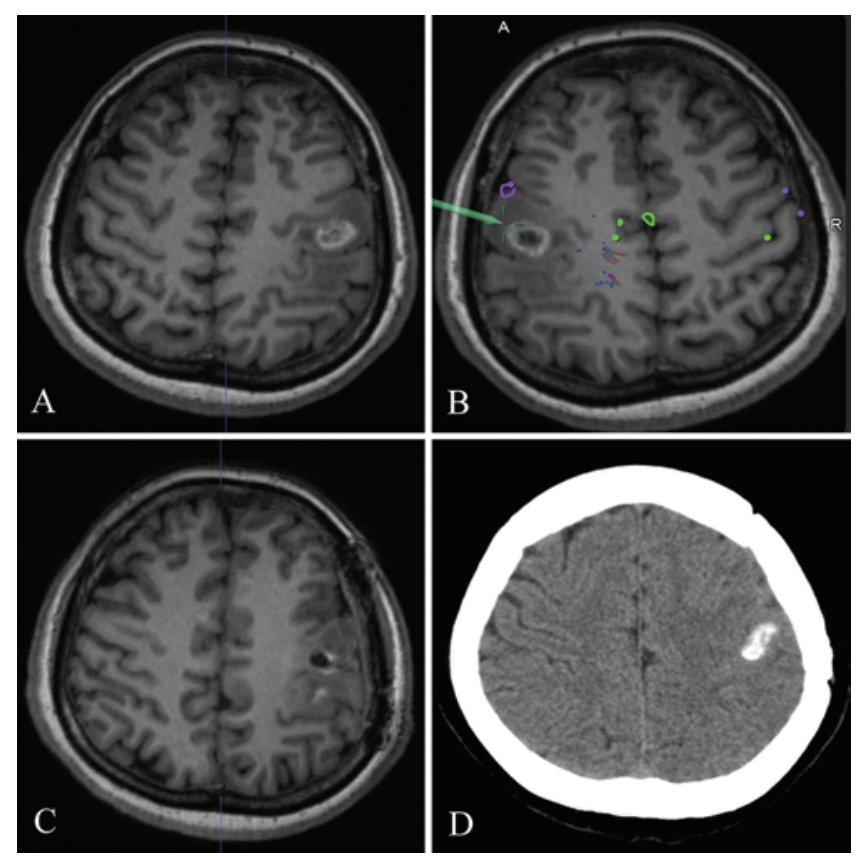

FIG. 5. A: A CM adjacent to the CST at the cortical level was detected on preoperative MRI in a female patient. B: Microsurgery and intraoperative DTI navigation were performed to remove the lesion. C: Postoperative MR image obtained 5 days after surgery, showing that the lesion was radically removed. D: However, 7 months later, the patient had a sudden onset of repeated seizures, and an emergent CT scan revealed an intracranial hemorrhage located at the prior surgical cavity. Figure is available in color online only.

patients with CMs involving the posterior limb of the internal capsule (Fig. 4), and the remaining 2 (12.5\%) lesions located at a lower level were removed by transgressing the noneloquent temporal cortex (Table 2). Complete resection of the CST-CM was attained in all but one (98.2\%) patient (Fig. 5). In this patient, a postoperative MRI scan obtained 5 days after surgery showed that the lesion was radically removed. However, 7 months later, the patient experienced a sudden onset of repeated seizures, and an emergent CT scan revealed an intracranial hemorrhage located at the prior surgical cavity. Positive sites of subcortical electric stimulation at the resection cavity walls were obtained in 50 (89.3\%) patients (Table 1). Four (7.2\%) patients had postoperative surgical complications, including $3(5.4 \%)$ patients who had an intracranial infection and $1(1.8 \%)$ patient who had a new seizure onset. However, the intracranial infections were resolved, and the seizure was controlled before discharge. One week after surgery, $21(37.5 \%)$ patients had short-term surgery-related motor deficits, but only 14 (25.0\%) patients had long-term motor deficits at the last clinical visit (at least 3 months after surgery). The mean patient follow-up was $14.7 \pm 10.1$ months, and the mean final $\mathrm{mRS}$ score was $1.1 \pm 1.2$. Details of the preoperative and final mRS scores are shown in Fig. 6. At the last clinical visit, $8(14.3 \%)$ patients had poor surgical outcome (final mRS score $>2$ ). However, only $1(1.8 \%)$ patient with a relatively short follow-up period (3 months) had unacceptable surgical outcomes (final mRS score of 4). Although the mean value of the mRS score seemed to improve at the final clinical visit, the difference between

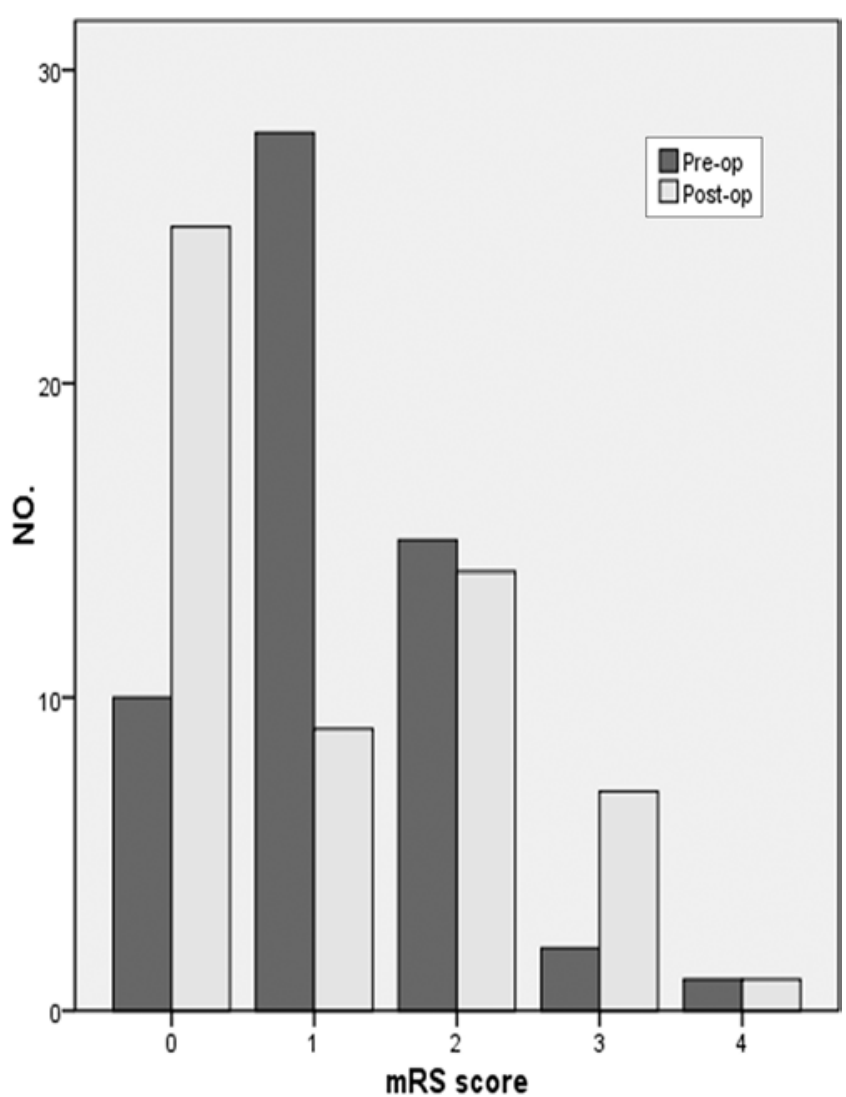

FIG. 6. The details of the preoperative mRS scores (black) and mRS scores at the last clinical visit (white) of the patients. The difference between the preoperative and final mRS scores was not statistically significant $(p=0.490)$.

preoperative and final $\mathrm{mRS}$ scores was not statistically significant $(\mathrm{p}=0.490)$ (Fig. 6).

\section{Risk Factors for Short-Term Surgery-Related Motor Deficits}

According to the univariate analysis, a short LCD (OR $0.54,95 \%$ CI $0.39-0.75 ; \mathrm{p}<0.001)$ and CST alteration (OR $4.50,95 \%$ CI $1.40-14.50 ; \mathrm{p}=0.012$ ) were significantly associated with short-term surgery-related motor deficits (Table 3). In addition, deep location of the CST-CM (OR $2.97,95 \%$ CI $0.93-9.44 ; p=0.065)$ had a trend toward significance on univariate analysis (Table 3 ). These 3 variables were included in the multivariate analysis, which revealed that independent factors that correlated with short-term surgery-related motor deficits were a short LCD (OR 0.52, 95\% CI 0.36-0.75; $\mathrm{p}<0.001)$ and CST alteration (OR 4.74, 95\% CI 1.00-22.33; $\mathrm{p}=0.049$ ) (Table 3). An ROC analysis was done to test the predictive value of the LCD for shotterm motor deficits. The area under the ROC curve (AUC) was 0.860 , and the cutoff point was $2.55 \mathrm{~mm}$ (Fig. 7).

\section{Risk Factors for Long-Term Surgery-Related Motor Deficits}

Based on the univariate analysis, a short LCD (OR 0.41, 95\% CI 0.24-0.70; $\mathrm{p}=0.001$ ), aDVA (OR 4.02, 95\% CI $1.12-14.36 ; \mathrm{p}=0.032$ ), and deep location of the CST-CM 
TABLE 3. Univariate and multivariate logistic regression analyses to test the association of each predictor with the surgery-related motor deficits at 7 days after surgery

\begin{tabular}{|c|c|c|c|c|c|c|}
\hline \multirow[b]{2}{*}{ Variable } & \multicolumn{3}{|c|}{ Univariate Logistic Regression } & \multicolumn{3}{|c|}{ Multivariate Logistic Regression } \\
\hline & OR & $95 \% \mathrm{Cl}$ & p Value & OR & $95 \% \mathrm{Cl}$ & $\mathrm{p}$ Value \\
\hline Sex & 1.54 & $0.49-4.84$ & 0.461 & & & \\
\hline Age & 1.01 & $0.97-1.05$ & 0.627 & & & \\
\hline No. of hemorrhages & 0.70 & $0.32-1.53$ & 0.366 & & & \\
\hline ILHS & 1.00 & $0.98-1.01$ & 0.666 & & & \\
\hline Previous radiosurgery & 0.83 & $0.07-9.69$ & 0.878 & & & \\
\hline Side & 0.86 & $0.29-2.54$ & 0.783 & & & \\
\hline Diameter & 1.02 & $0.97-1.07$ & 0.473 & & & \\
\hline Location & 2.97 & $0.93-9.44$ & 0.065 & 1.64 & $0.36-7.44$ & 0.522 \\
\hline Hematoma & 1.34 & $0.43-4.17$ & 0.610 & & & \\
\hline Edema & 0.60 & $0.18-2.04$ & 0.411 & & & \\
\hline Multiple CMs & 1.29 & $0.26-6.43$ & 0.755 & & & \\
\hline aDVA & 2.40 & $0.79-7.32$ & 0.124 & & & \\
\hline Preop mRS score & 1.06 & $0.56-2.01$ & 0.869 & & & \\
\hline Preop MD & 1.16 & $0.34-3.89$ & 0.815 & & & \\
\hline LCD & 0.54 & $0.39-0.75$ & $<0.001$ & 0.52 & $0.36-0.75$ & $<0.001$ \\
\hline CST alteration & 4.50 & $1.40-14.50$ & 0.012 & 4.74 & $1.00-22.33$ & 0.049 \\
\hline Complications & 1.74 & $0.23-13.35$ & 0.596 & & & \\
\hline
\end{tabular}

Boldface type indicates statistical significance or a trend toward significance for location.

(OR 7.26, 95\% CI 1.44-36.54; $\mathrm{p}=0.016$ ) were significantly associated with long-term surgery-related motor deficits (Table 4). CST alteration (OR 3.33, 95\% CI 0.95-11.66; $\mathrm{p}=$ $0.060)$ trended toward significance on univariate analysis (Table 4). All significant variables and CST alteration were included in the multivariate analysis. Multivariate analysis revealed that a short LCD (OR 0.39, 95\% CI 0.22-0.70; p

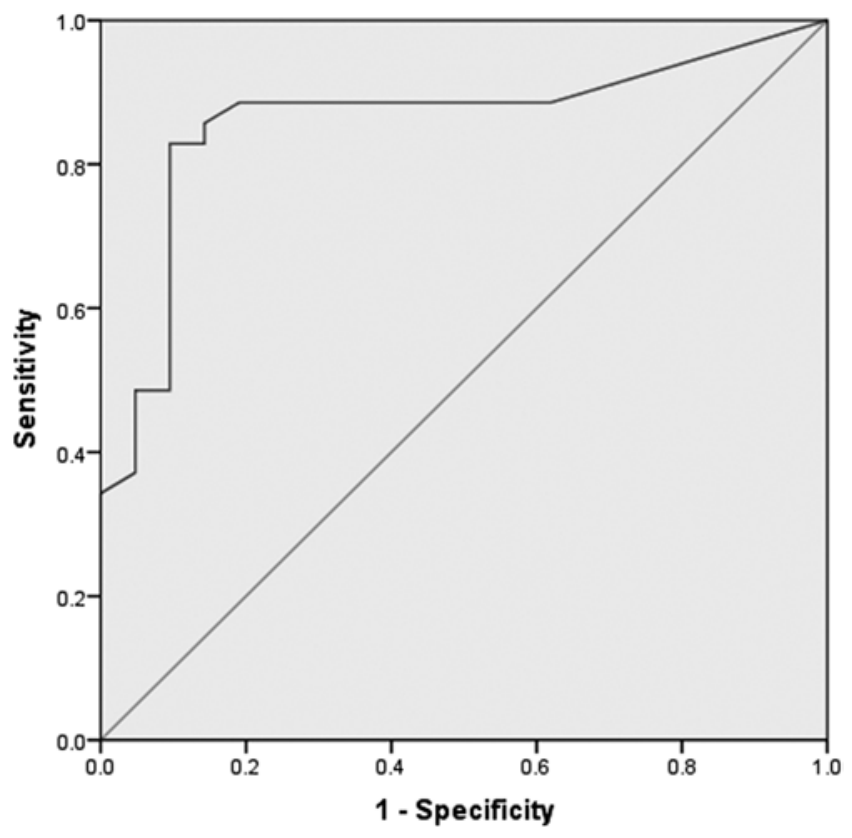

FIG. 7. The ROC curve analysis for the relationship between LCD and short-term surgery-related motor deficits. The AUC was 0.860 , and the cutoff point was $L C D=2.55 \mathrm{~mm}$.
$=0.002)$ was the only independent factor that correlated with long-term motor deficits. Deep location of a CST-CM (OR 6.87, 95\% CI 0.92-51.26; $\mathrm{p}=0.060$ ) had a trend toward significance on multivariate analysis (Table 4). An ROC curve analysis was done to test the predictive value of the LCD for long-term surgery-related motor deficits. The AUC was 0.894 , and the cutoff point was $2.30 \mathrm{~mm}$ (Fig. 8).

\section{Discussion}

\section{LCD and Surgery-Related Motor Deficits}

In this study, both short-term $(\mathrm{p}<0.001)$ and long-term $(p=0.002)$ surgery-related motor deficits were significantly associated with LCD (Tables 3 and 4). ROC curve analysis was as follows: for short-term motor deficits, the AUC was 0.860 , and the cutoff point was $\mathrm{LCD}=2.55 \mathrm{~mm}$; for long-term motor deficits, the AUC was 0.894, and the cutoff point was $L C D=2.30 \mathrm{~mm}$. Krishnan et al. investigated the relationship between the lesion-to-motor activation distance on preoperative functional MRI and new-onset motor deficits after surgery in 54 patients with rolandic tumors. ${ }^{19}$ The authors advocated that a lesion-to-activation distance of less than $5 \mathrm{~mm}$ may be associated with a higher risk of postoperative neurological deterioration. In our published study, we aimed to determine the association between functional findings and worsened muscle strength in patients harboring an AVM in a motor eloquent area. ${ }^{26}$ Results of statistical analysis showed that in patients with an LCD of 0-5 mm, surgical treatment seemed to have a high risk of transient muscle strength worsening, and a meticulous technique should be performed to protect the CST during surgery. In this study, the LCD was defined 
TABLE 4. Univariate and multivariate logistic regression analyses to test the association of each predictor with the surgery-related motor deficits at the last clinical visit

\begin{tabular}{|c|c|c|c|c|c|c|}
\hline \multirow[b]{2}{*}{ Variable } & \multicolumn{3}{|c|}{ Univariate Logistic Regression } & \multicolumn{3}{|c|}{ Multivariate Logistic Regression } \\
\hline & OR & $95 \% \mathrm{Cl}$ & $p$ Value & OR & $95 \% \mathrm{Cl}$ & $\mathrm{p}$ Value \\
\hline Sex & 0.80 & $0.21-3.01$ & 0.741 & & & \\
\hline Age & 1.01 & $0.96-1.07$ & 0.612 & & & \\
\hline No. of hemorrhages & 0.04 & $0.26-1.60$ & 0.339 & & & \\
\hline ILHS & 1.01 & $0.99-1.02$ & 0.383 & & & \\
\hline Previous radiosurgery & 1.54 & $0.13-18.38$ & 0.734 & & & \\
\hline Side & 0.68 & $0.20-2.31$ & 0.538 & & & \\
\hline Diameter & 1.04 & $0.99-1.10$ & 0.135 & & & \\
\hline Location & 7.26 & $1.44-36.54$ & 0.016 & 6.87 & $0.92-51.26$ & 0.06 \\
\hline Hematoma & 0.72 & $0.12-2.70$ & 0.626 & & & \\
\hline Edema & 0.55 & $0.13-2.28$ & 0.406 & & & \\
\hline Multiple CMs & 0.46 & $0.05-4.21$ & 0.493 & & & \\
\hline aDVA & 4.02 & $1.12-14.36$ & 0.032 & 1.31 & $0.11-15.99$ & 0.831 \\
\hline Preop mRS & 1.14 & $0.56-2.33$ & 0.713 & & & \\
\hline Preop MD & 1.78 & $0.48-6.55$ & 0.387 & & & \\
\hline LCD & 0.41 & $0.24-0.70$ & 0.001 & 0.39 & $0.22-0.70$ & 0.002 \\
\hline CST alteration & 3.33 & $0.95-11.66$ & 0.060 & 2.82 & $0.44-18.06$ & 0.274 \\
\hline Complications & 1.00 & $0.09-10.47$ & 1.000 & & & \\
\hline Follow-up & 1.03 & $0.97-1.09$ & 0.318 & & & \\
\hline
\end{tabular}

Boldface type indicates statistical significance.

as the distance between the tracked CST and the margin of the CM on sagittal T1-weighted anatomical images, but not to the margins of the hematoma or hemosiderin rim (Figs. 1 and 2). Because the hematoma was evacuated and the hemosiderin rim was preserved during surgery, only the vascular malformation needed to be separated from the surrounding brain tissue. According to our results, the safe LCD was shorter in patients with CMs than that in patients with tumors or AVMs ( $5 \mathrm{~mm}$ for tumors or AVMs vs 2.55 $\mathrm{mm}$ for CMs). In the context of brain tumor, lesions can infiltrate into the brain tissue, which may force the neurosurgeon to establish dissection planes that may extend into eloquent brain tissues. ${ }^{4,28,36}$ However, CMs are histologically benign vascular lesions consisting of thin-walled sinusoidal vascular channels without interposed brain tissue, and the boundaries are clear in most cases..$^{35}$ Thus, operative trauma to the adjacent brain tissue is less likely to occur than that in patients with tumors. The longer safe LCD $(5 \mathrm{~mm})$ in patients with an AVM may be attributed to the angioarchitecture features. AVMs have numerous feeding arteries, and achieving hemostasis of the dissecting plane may be quite difficult, especially for feeding arteries from perforating arteries, which are thin, fragile, and difficult to occlude with cautery. ${ }^{12}$ The hemostasis process may cause severe trauma to the surrounding brain tissues. According to our results, and to facilitate clinical practice, we propose that $3 \mathrm{~mm}(2.55$ to 3.00$)$ may be the safe LCD for surgery in patients with $\mathrm{CM}$.

\section{CST Alteration and Surgery-Related Motor Deficits}

Both univariate $(\mathrm{p}=0.012)$ and multivariate $(\mathrm{p}=0.049)$ analyses revealed that CST alteration on preoperative DTI was significantly correlated with short-term surgery-related motor deficits (Table 3). Furthermore, CST alteration had a trend toward significance with long-term motor deficits on univariate analysis $(\mathrm{p}=0.06)$ (Table 4). The feasibility and utility of DTI tractography has been determined in various intracranial lesions, ${ }^{4,18,22,28}$ including in the con-

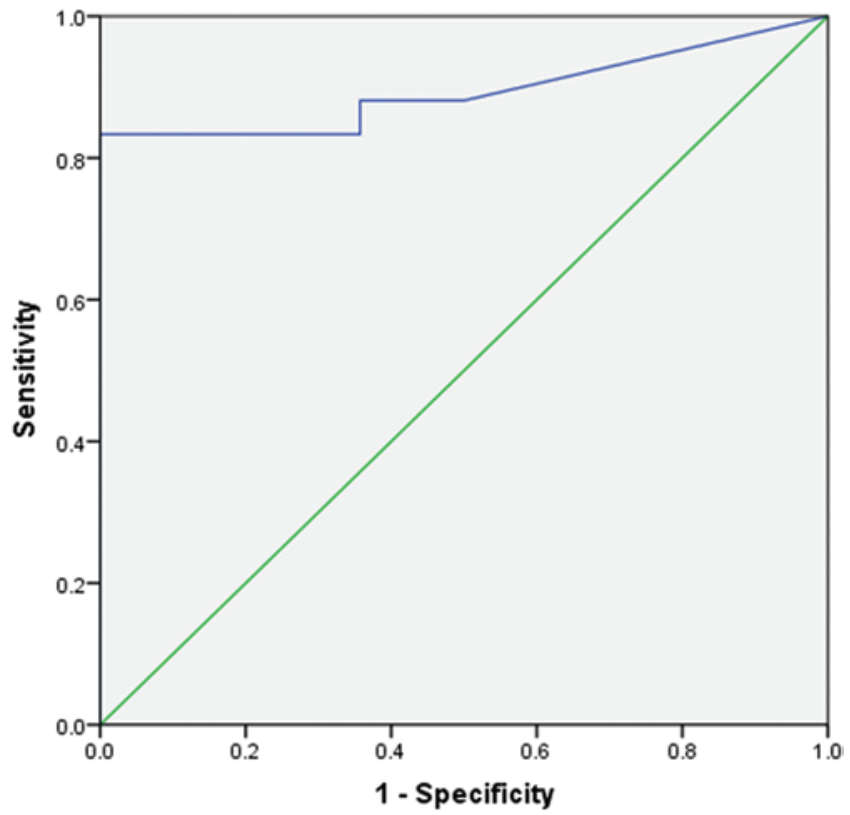

FIG. 8. The ROC curve analysis for the relationship between LCD and long-term surgery-related motor deficits. The AUC was 0.894 , and the cutoff point was $L C D=2.30 \mathrm{~mm}$. Figure is available in color online only. 
text of CMs. ${ }^{8,24}$ Recently, Cauley et al. ${ }^{8}$ proposed that the hemosiderin rims of CMs were intimately associated with white matter tracts that deviated because of the central lesion. These findings are consistent with histopathological reports that the hemosiderin rim is composed of blood breakdown products deposited in viable white matter. ${ }^{32,34}$ Thus, the CST could pass through the hemosiderin rim and come in contact with the vascular malformation or the hematoma, causing various alterations of the CST on preoperative DTI. As concluded by Bérubé et al., ${ }^{5}$ alterations of the CST on preoperative DTI can be caused by lesion mass effect, change in the number of the axons, change in the myelin sheath surrounding axons, or change in the organization/direction of the fibers. Therefore, many authors have come to the conclusion that CST alteration may correlate with preoperative functional disorder and the risk of postoperative deficits. ${ }^{5,20}$ In our previous study about patients with CMs involving the posterior limb of the internal capsule ${ }^{24}$ we used an involvement grading system of the major fiber tracts to show that findings on preoperative DTI studies were significantly correlated with surgical outcomes. The aforementioned opinions were consistent with the results of this study. CST alteration on preoperative DTI studies meant that the CST had been involved or compressed by the lesion, and resection would have a higher risk of postoperative motor deficits.

\section{Other Risk Factors for Surgery-Related Motor Deficits}

On univariate analysis, deep location of the CST-CMs had a trend toward significance with short-term surgeryrelated motor deficits $(\mathrm{p}=0.065)$, but was significantly correlated with long-term $(\mathrm{p}=0.016)$. Furthermore, deep location of the CST-CMs also had a trend toward significance with long-term motor deficits in the multivariate analysis $(\mathrm{p}=0.060)$ (Tables 3 and 4). In this study, CMs adjacent to the CST at the corona radiata level were defined as deep CST-CMs because most involved critical deep structures, such as the paraventricular parenchyma, insular lobe, and basal ganglia (Figs. 2 and 4). In patients with deep CST-CMs, a long access was needed to approach the CST-CMs (Fig. 4). Transgression of brain parenchyma, a wide opening of the sulcus or sylvian fissure, and skeletonization of the nearby vessels may cause iatrogenic damage to eloquent brain tissues. ${ }^{16,17,21,23,29-31}$ DTI is useful for appreciating the complex relationships between critical white matter structures and the anatomical location of the deep CST-CMs. The unique capabilities of DTI tractography for localizing, segmenting, and mapping fiber tracts make it a promising method for visualizing the relationship between the CST and the deep-seated CMs. ${ }^{20,24,27}$ Furthermore, the other variable that might be associated with surgery-related motor deficits is an aDVA. In this study, aDVA was detected in 22 (39.3\%) patients on preoperative MRI studies. Univariate analysis showed that aDVA was significantly correlated with long-term motor deficits. Benign natural history $(0.15 \%-0.34 \%$ symptomatic annual hemorrhage rate), coupled with the role in draining the normal parenchyma, made aDVA an important influence on the selected surgical approach and surgical outcomes..$^{17,30}$ Thus, their disruption must be avoided to prevent a potentially fatal venous infarction.

\section{Surgery for CST-CMs}

In this study, all CST-CMs were surgically treated, and a complete resection was archived in $55(98.2 \%)$ patients. At the last clinical visit, only $8(14.3 \%)$ patients had poor surgical outcome (final mRS score $>2$ ). The difference between preoperative and final mRS scores was not statistically significant $(\mathrm{p}=0.490)$ (Fig. 6). There is general agreement in the literature regarding the surgical treatment for symptomatic supratentorial CMs located in noneloquent areas. ${ }^{21,30}$ However, some controversy exists about managing supratentorial CMs located in eloquent or deep areas..$^{11,35}$ Their management can be conservative or include microsurgical resection or radiosurgery, with the indications for their resection being controversial. ${ }^{1}$ Considering the technical difficulty of performing surgery next to the CST and the significant associated operative risks, conservative management is often recommended for patients. Unfortunately, the yearly bleeding incidence without surgical intervention was reported to be $2.4 \%$ for incidental lesions and $29.5 \%$ for symptomatic CMs. ${ }^{3}$ Such repetitive microbleeding may lead to progressive neurological symptoms or seizure in up to $16.5 \%$ of patients per year. ${ }^{33}$ Therefore, outcomes after conservative treatment might be worse than those after microsurgery. ${ }^{23}$ On the other hand, as we mentioned before, radiosurgery at our institution was not an alternative treatment method for patients with CMs. We agree that radiosurgery requires further study, considering the lack of histological change, latency required before efficacy, and the potential for radiation-induced morbidity. ${ }^{2}$ Recently, the widespread use of modern state-of-the-art techniques, including functional MRI, DTI, neuronavigation, and neuromonitoring, renders surgery of CMs in critical areas safer. ${ }^{35}$ The risk of long-term surgical morbidity caused by CMs in critical areas has been recently reported to be between $10 \%$ and 20\%. ${ }^{29,30}$ Our surgical outcomes of patients with CSTCMs were comparable to those in the literature: $14.3 \%$ patients had poor surgical outcomes (final mRS score $>2$ ), and only $1(1.8 \%)$ patient with a relatively short follow-up period (3 months) had an unacceptable surgical outcome (final mRS score of 4). However, patient selection remains a critical determinant of results, and it has not been addressed in the literature. In this study, we identified the risk factors for surgery-related motor deficits and determined the prognostic effect of preoperative DTI tractography in patients with CST-CM. Based on the results of our statistical analysis, we offer the following suggestions to facilitate clinical decision making in treating CST-CMs: 1) In patients with an LCD $\geq 3 \mathrm{~mm}$, surgery is recommended with a low risk of surgery-related motor deficits. 2) In patients with an LCD $<3 \mathrm{~mm}$ and CST alterations, surgery has a high risk of surgery-related motor deficits at 7 days after surgery. 3) In patients with an LCD $<3 \mathrm{~mm}$ and a deep location, surgery has a high risk of long-term motor deficits. 4) Preoperative DTI tractography and detecting aDVA are necessary to improve surgical outcomes.

\section{Limitations}

Although DTI is effective, it remains a model-based technique, and, as a consequence of its reliance on a tensor, it could be influenced by surrounding edema and as- 
sociated hematoma. ${ }^{13,14}$ In this study, the ipsilesional CST was not tracked in one patient who had a short interval between last hemorrhage and surgery, with obvious edema and a large hematoma surrounding the CST-CM. Furthermore, patients at our hospital were screened in the outpatient department. The only patients admitted to the neurosurgical department were those who needed surgery. Thus, the natural history of untreated CST-CMs cannot be determined, and the difference in the outcomes between surgically treated and conservatively observed patients cannot be documented. In addition, this study is limited by not having a control group of patients without preoperative DTI. Thus, we cannot determine the effect of preoperative planning with DTI on surgical outcomes in patients with CST-CM in this study. However, we identified the risk factors for motor deficits, and determined the prognostic effect of preoperative DTI findings, which may facilitate clinical decision making in patients with CST-CM.

\section{Conclusions}

To facilitate clinical practice, we propose that $3.00 \mathrm{~mm}$ (2.55 to $\sim 3.00 \mathrm{~mm}$ ) may be the safe LCD for surgery in patients with CST-CMs. Moreover, a CST alteration on preoperative DTI and a deep location of the CST-CM may be the factors for short-term and long-term surgery-related motor deficits, respectively. A randomized controlled trial is needed to evaluate the effect of preoperative planning with DTI on surgical outcomes in patients with CST-CM in our future studies.

\section{Acknowledgments}

This study was supported by a National Science and Technology Support Plan (No. 2011BAI08B08, principal investigator: Professor Shuo Wang), a grant from the Ministry of Health of China, and by the 973 National Key Basic Research Development Plan (No. 2012CB720704, principal investigator: Professor Zhen Jin) grant from the Ministry of Science and Technology of China.

\section{References}

1. Abhinav K, Pathak S, Richardson RM, Engh J, Gardner P, Yeh FC, et al: Application of high-definition fiber tractography in the management of supratentorial cavernous malformations: a combined qualitative and quantitative approach. Neurosurgery 74:668-681, 2014

2. Abla AA, Spetzler RF: Cavernous malformations of the thalamus: a relatively rare but controversial entity. World Neurosurg 79:641-644, 2013

3. Al-Shahi Salman R, Hall JM, Horne MA, Moultrie F, Josephson CB, Bhattacharya JJ, et al: Untreated clinical course of cerebral cavernous malformations: a prospective, populationbased cohort study. Lancet Neurol 11:217-224, 2012

4. Bello L, Gambini A, Castellano A, Carrabba G, Acerbi F, Fava E, et al: Motor and language DTI fiber tracking combined with intraoperative subcortical mapping for surgical removal of gliomas. Neuroimage 39:369-382, 2008

5. Bérubé J, McLaughlin N, Bourgouin P, Beaudoin G, Bojanowski MW: Diffusion tensor imaging analysis of long association bundles in the presence of an arteriovenous malformation. J Neurosurg 107:509-514, 2007

6. Byrnes TJD, Barrick TR, Bell BA, Clark CA: Semiautomatic tractography: motor pathway segmentation in patients with intracranial vascular malformations. Clinical article. J Neurosurg 111:132-140, 2009
7. Catani M, Thiebaut de Schotten M: A diffusion tensor imaging tractography atlas for virtual in vivo dissections. Cortex 44:1105-1132, 2008

8. Cauley KA, Andrews T, Gonyea JV, Filippi CG: Magnetic resonance diffusion tensor imaging and tractography of intracranial cavernous malformations: preliminary observations and characterization of the hemosiderin rim. J Neurosurg 112:814-823, 2010

9. Chang EF, Gabriel RA, Potts MB, Berger MS, Lawton MT: Supratentorial cavernous malformations in eloquent and deep locations: surgical approaches and outcomes. Clinical article. J Neurosurg 114:814-827, 2011

10. Conway JE, Rigamonti D: Cavernous malformations: a review and current controversies. Neurosurg Q 16:15-23, 2006

11. D'Angelo VA, De Bonis C, Amoroso R, Cali A, D'Agruma L, Guarnieri V, et al: Supratentorial cerebral cavernous malformations: clinical, surgical, and genetic involvement. Neurosurg Focus 21(1):e9, 2006

12. Du R, Keyoung HM, Dowd CF, Young WL, Lawton MT: The effects of diffuseness and deep perforating artery supply on outcomes after microsurgical resection of brain arteriovenous malformations. Neurosurgery 60:638-648, 2007

13. Duffau $\mathrm{H}$ : The dangers of magnetic resonance imaging diffusion tensor tractography in brain surgery. World Neurosurg 81:56-58, 2014

14. Duffau H: Diffusion tensor imaging is a research and educational tool, but not yet a clinical tool. World Neurosurg 82:e43-e45, 2014

15. Flores BC, Whittemore AR, Samson DS, Barnett SL: The utility of preoperative diffusion tensor imaging in the surgical management of brainstem cavernous malformations. J Neurosurg 122:653-662, 2015

16. Garcia RM, Ivan ME, Lawton MT: Brainstem cavernous malformations: surgical results in 104 patients and a proposed grading system to predict neurological outcomes. Neurosurgery 76:265-278, 2015

17. Gross BA, Batjer HH, Awad IA, Bendok BR: Cavernous malformations of the basal ganglia and thalamus. Neurosurgery 65:7-19, 2009

18. Itoh D, Aoki S, Maruyama K, Masutani Y, Mori H, Masumoto T, et al: Corticospinal tracts by diffusion tensor tractography in patients with arteriovenous malformations. J Comput Assist Tomogr 30:618-623, 2006

19. Krishnan R, Raabe A, Hattingen E, Szelényi A, Yahya H, Hermann E, et al: Functional magnetic resonance imagingintegrated neuronavigation: correlation between lesion-tomotor cortex distance and outcome. Neurosurgery 55:904915,2004

20. Lazar M, Alexander AL, Thottakara PJ, Badie B, Field AS: White matter reorganization after surgical resection of brain tumors and vascular malformations. AJNR Am J Neuroradiol 27:1258-1271, 2006

21. Leal PRL, Houtteville JP, Etard O, Emery E: Surgical strategy for insular cavernomas. Acta Neurochir (Wien) 152:1653-1659, 2010

22. Lee JS, Han MK, Kim SH, Kwon OK, Kim JH: Fiber tracking by diffusion tensor imaging in corticospinal tract stroke: topographical correlation with clinical symptoms. Neuroimage 26:771-776, 2005

23. Li D, Zhang J, Hao S, Tang J, Xiao X, Wu Z, et al: Surgical treatment and long-term outcomes of thalamic cavernous malformations. World Neurosurg 79:704-713, 2013

24. Lin F, Wu J, Wang L, Zhao B, Tong X, Jin Z, et al: Surgical treatment of cavernous malformations involving the posterior limb of the internal capsule: utility and predictive value of preoperative diffusion tensor imaging. World Neurosurg 88:538-547, 2016

25. Lin F, Wu J, Zhao B, Tong X, Jin Z, Cao Y, et al: Preopera- 
tive functional findings and surgical outcomes in patients with motor cortical arteriovenous malformation. World Neurosurg 85:273-281, 2016

26. Lin F, Zhao B, Wu J, Wang L, Jin Z, Cao Y et al: Risk factors for worsened muscle strength after the surgical treatment of arteriovenous malformations of the eloquent motor area. J Neurosurg 125:289-298, 2016

27. Niizuma K, Fujimura M, Kumabe T, Higano S, Tominaga T: Surgical treatment of paraventricular cavernous angioma: fibre tracking for visualizing the corticospinal tract and determining surgical approach. J Clin Neurosci 13:1028-1032, 2006

28. Okada T, Mikuni N, Miki Y, Kikuta K, Urayama S, Hanakawa T, et al: Corticospinal tract localization: integration of diffusion-tensor tractography at 3-T MR imaging with intraoperative white matter stimulation mapping-preliminary results. Radiology 240:849-857, 2006

29. Pandey P, Westbroek EM, Gooderham PA, Steinberg GK: Cavernous malformation of brainstem, thalamus, and basal ganglia: a series of 176 patients. Neurosurgery 72:573-589, 2013

30. Pasqualin A, Meneghelli P, Giammarusti A, Turazzi S: Results of surgery for cavernomas in critical supratentorial areas. Acta Neurochir Suppl 119:117-123, 2014

31. Rangel-Castilla L, Spetzler RF: The 6 thalamic regions: surgical approaches to thalamic cavernous malformations, operative results, and clinical outcomes. J Neurosurg 123:676-685, 2015

32. Tomlinson FH, Houser OW, Scheithauer BW, Sundt TM Jr, Okazaki H, Parisi JE: Angiographically occult vascular malformations: a correlative study of features on magnetic resonance imaging and histological examination. Neurosurgery 34:792-800, 1994

33. Washington CW, McCoy KE, Zipfel GJ: Update on the natural history of cavernous malformations and factors predicting aggressive clinical presentation. Neurosurg Focus 29(3):E7, 2010

34. Wong JH, Awad IA, Kim JH: Ultrastructural pathological features of cerebrovascular malformations: a preliminary report. Neurosurgery 46:1454-1459, 2000

35. Wostrack M, Shiban E, Harmening K, Obermueller T, Ringel F, Ryang YM, et al: Surgical treatment of symptomatic cerebral cavernous malformations in eloquent brain regions. Acta Neurochir (Wien) 154:1419-1430, 2012

36. Zolal A, Hejčl A, Vachata P, Bartoš R, Humhej I, Malucelli A, et al: The use of diffusion tensor images of the corticospinal tract in intrinsic brain tumor surgery: a comparison with direct subcortical stimulation. Neurosurgery 71:331-340, 2012

\section{Disclosures}

The authors report no conflict of interest concerning the materials or methods used in this study or the findings specified in this paper.

\section{Author Contributions}

Conception and design: Y Lin, F Lin, Cao. Acquisition of data: Y Lin, F Lin, Jiao. Analysis and interpretation of data: Y Lin, F Lin. Drafting the article: Y Lin, F Lin. Critically revising the article: Wang, F Lin, Kang. Reviewed submitted version of manuscript: all authors. Approved the final version of the manuscript on behalf of all authors: Wang. Statistical analysis: Y Lin, F Lin. Administrative/technical/material support: Wang, Kang, Cao. Study supervision: Wang, Cao.

\section{Correspondence}

Shuo Wang, Beijing Tiantan Hospital, Capital Medical University, Department of Neurosurgery, No. 6 Tiantanxili, Dongcheng district, Beijing 100050, China. email: captainwang9858@126.com. 